\title{
Opfer von Menschenhandel zum Zweck der sexuellen Ausbeutung - Verläufe von Viktimisierung und Voraus- setzungen für die Kooperationsbereitschaft mit der Polizei
}

Cornelia Helfferich, Barbara Kavemann

\section{Einleitung}

Die Bereitschaft der Opfer von Menschenhandel, eine Aussage bei der Polizei zu machen, ist die wichtigste Voraussetzung für eine gelingende Strafverfolgung der Taten nach $\mathbb{2} 232$ StGB, unabhängig davon, wie der Kontakt der Betroffenen mit der Polizei zustande kommt. In 2009 resultierten 303 (57\%) der eingeleiteten Verfahren aus polizeilichen Maßnahmen. Immerhin gut ein Drittel (181 Verfahren, 34 \%) wurden aufgrund von Anzeigen der Opfer eingeleitet (BKA 2009). Die Kooperationsbereitschaft der Betroffenen gewinnt im Zuge der EUErweiterung an Bedeutung, denn die Mehrheit der Migrantinnen, die Opfer von Menschenhandel werden, stammt inzwischen aus EU-Mitgliedsstaaten und verfügt über legale Aufenthaltsmöglichkeiten.

Über Menschenhandel liegen wenig systematisch erhobene Forschungsergebnisse vor, die Datenlage entspricht nicht dem Maß an politischer und medialer Aufmerksamkeit, die der Thematik zuteil wird. Es gibt keine verlässlichen Erkenntnisse über das Ausmaß von Menschenhandel, was für Straftaten im Umfeld organisierter Kriminalität typisch ist (Goodey 2008). Die Identifikation von Betroffenen und ihre Vernehmung als Zeuginnen gelingt nach Annahme einiger Autor/innen eher selten (Gallagher / Holmes 2008). Forschung befasste sich mit Fragen der Strafverfolgung (Herz/Minthe 2005), Täterstrategien wurden ausschließlich im Zusammenhang mit der Logistik organisierter Kriminalität erforscht (Sieber / Bögel 1993), die jedoch einen eher kleinen Anteil des Menschenhandels zum Zweck der sexuellen Ausbeutung ausmacht (van Duyne / Lamp / Passas 2002). Bisherige Opferbefragungen (Baurmann/Schädler 1999 / Voß 2001 / Balß u.a. 2001 / Schädler 2002) konzentrierten sich auf den polizeilichen Umgang mit Opfern von Straftaten, ohne die Aussagebereitschaft der Opfer in den Blickpunkt zu rücken. Die hier vorgestellte Studie $^{1}$ nimmt explizit die Motive und die subjektive Perspektive der Betroffenen in den Blick.
Die hier präsentierte Studie stellt mit einer Stichprobe von 53 Frauen, die Opfer von Menschenhandel zum Zweck der sexuellen

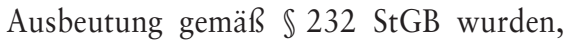
eine vergleichsweise umfangreiche Opferbefragung dar. Frauen zwischen 21 und 41 Jahren aus 19 Ländern in sehr unterschiedlichen Lebenssituationen konnten in teilnarrativen Interviews befragt werden. Darunter waren 37 Frauen, die als Zeuginnen in Ermittlungsverfahren ausgesagt hatten, elf Frauen, die zwar Kontakt mit der Polizei hatten, sich aber nicht zu einer Aussage entschlossen und fünf Frauen, die keinen Kontakt mit der Polizei hatten und diesen auch nicht wollten. Die zentrale Forschungsfrage lautete: Welche Variablen beeinflussen die Aussagebereitschaft der Opfer (bei der Polizei bzw. bei Fachberatungsstellen) im Sinne von Risiko- und Erfolgsfaktoren für ein Gewinnen von Aussagebereitschaft? Erhoben wurden die subjektiven Sichtweisen der Frauen. Aus den Interviews wurden Determinanten der Aussagebereitschaft in ihrer Bedeutung für polizeiliches Handeln, aber auch polizeiliches Handeln selbst als Determinante $^{2}$ der Aussagebereitschaft rekonstruiert. In einem weiteren Schritt wurden mehrere typische Abläufe von Viktimisierungsverläufen rekonstruiert

Die Studie zeigt, dass vorschnelle Verallgemeinerungen auf alle Betroffenen von Menschenhandel zum Zweck der sexuellen Ausbeutung ebenso wenig möglich sind, wie die Identifikation einer einzelnen Determinante als allgemeiner Schlüssel zur Aussagebereitschaft. Die Gruppe der Opfer ist heterogen, die Viktimisierungsverläufe sehr unterschiedlich und die Determinanten bilden komplexe Zusammenhänge.

\section{Die Bedeutung einzel-} ner Determinanten der Aussagebereitschaft

Eine Reihe angenommener Determinanten der Aussagebereitschaft wurde in ihrer Bedeutung bestätigt. Im Rahmen einer inhaltsanalytischen Querauswertung wurden sie in ihrer subjektiven Bedeutung induktiv aus dem Material heraus ergänzt und binnendifferenziert sowie in ihrer Stärke bestimmt. Die Determinanten hatten drei Funktionen im Geschehen: Sie konnten als - überwiegend starke - Kräfte wirken, die Frauen in der Zwangssituation festhielten (Angst, Bindung), sie konnten Frauen aus der Situation „heraustreiben“ (Push-Faktoren z.B. Empörung oder auch Todesangst) und sie konnten Frauen in eine neue Situation „hineinziehen“ (Pull-Faktoren; z.B. Perspektiven oder Angebote für eine bessere Zukunft). Je nach Fallkontext konnte die Funktion und Bedeutung einer Determinante unterschiedlich sein.

Die identifizierten Determinanten ${ }^{3}$ lassen sich zum größten Teil vier Bereichen zuordnen:

a) Täterstrategien: Hier überwogen Strategien der Bedrohung und Gewalt. Die Angst vor den Täter/innen war ein wichtiger Faktor, der sowohl eine Aussage verhindern als auch die Aussage als einzigen Ausweg erscheinen lassen konnte. Relevant waren auch Strategien von Bindung durch Liebe und Ehe sowie, vor allem bei jungen Frauen, das Beeindrucken durch Statussymbole. Die von Bindungsstrategien betroffenen Frauen mussten sich aus der Beziehung lösen und sie als ein Zwangs- und Ausbeutungsverhältnis erkennen, bevor sie eine Aussagebereitschaft entwickeln konnten. Eine weitere relevante Täterstrategie war die „Schuldenfalle“ als Zwang, hohe Geldsummen „abzuarbeiten“, ohne ausreichenden Verdienst zugestanden zu bekommen.

b) Mit der Polizei und polizeilichem Handeln verbundene Determinanten: Negative Vorannahmen gegenüber und Angst vor der Polizei oder der Eindruck, die Polizei arbeite mit den Täter/innen zusammen, waren ein gewichtiges Motiv gegen die Entscheidung, sich an die Polizei zu wenden oder in Kontrollsituationen zu offenbaren.

c) Mit der Person verbundene Determinanten: Für den großen Teil der Frauen, 
die aus dem Ausland hierher gekommen oder gebracht worden waren, hatte das Erreichen ihres Migrationsziels - meist die Sicherung des Lebensunterhalts eine hohe Bedeutung. Relevant war auch die Einstellung zur Prostitution. Scham wirkte als Offenbarungshindernis, eine stark negative Einstellung zur Prostitution machte die erzwungene Ausübung der Prostitution psychisch zu einer extremen Belastung.

d) Rabmenbedingungen: Das Erreichen des Migrationsziels war gekoppelt an den rechtlichen Status. Vor allem Frauen aus Drittstaaten standen vor dem Problem, keinen rechtmäßigen Aufenthalt zu haben. Unkenntnis der Sprache, Schrift und Rechtslage behinderten die eigenständige Handlungsfähigkeit der Frauen. Auch Frauen mit legalem Aufenthaltsstatus hatten zum Teil keine verlässliche Kenntnis von ihrer rechtlichen Situation.

Die Determinanten bilden ein komplexes Gefüge. Täter/innen hatten z.B. durch den prekären rechtlichen Status von Opfern - sei es der objektive oder der subjektive Legalstatus - und die migrationsbedingten Barrieren, die eine Abhängigkeit der Betroffenen von den Täter/innen verfestigten, besondere und im Sinne der Täterintentionen günstige Bedingungen, Druck auszuüben. Umgekehrt verhinderte bzw. erschwerte dieser Status, dass Frauen sich zur Wehr setzen, sich informieren oder Zugang zu Unterstützung finden konnten.

Keine Determinante war allein für oder gegen eine Aussage entscheidend. Entsprechend den komplexen Problemlagen, in denen die Frauen sich befanden, waren die Einflussfaktoren widersprüchlich miteinander verbunden. Alle Motive konnten je nach Fallkonstellation die Aussagebereitschaft fördern oder hindern, alle Motive konnten zeitweilig oder dauerhaft für oder gegen eine Aussage sprechen.

\section{Polizeiliche Handlungsmöglichkeiten}

Das Spektrum, welche Organisationseinheit der Polizei bei welcher Gelegenheit auf welche Frauen traf, ist breit. Den einen Pol repräsentierte die Interviewpartnerin, die im Zuge ihrer Migration durch massive körperliche Gewalt in die Prostitution gezwungen wurde, die Prostitution zu keinem Zeitpunkt akzeptierte, vor den Täter/innen zur Polizei flüchtete und die Polizei als helfende Instanz adressierte. Der andere Pol wurde repräsen- tiert von einer Interviewpartnerin, die ihre Migration nach Deutschland gezielt in die Prostitution organisiert hatte, finanzielle Absprachen mit den Täter/innen als fair und die polizeiliche Intervention als Eingriff in ihre Freiheitsrechte interpretierte.

Die aus den Interviews rekonstruierte Interaktion zwischen den Interviewpartnerinnen und den Beamten/Beamtinnen der Polizei zeigte Anlässe dafür, dass die Strafverfolgungsbehörden einen Teil der Frauen bereits bei der Anzeigenaufnahme als Zeuginnen zunächst „verloren“ hatten bzw. Frauen nur eine Teilaussage (z.B. wegen häuslicher Gewalt) machten:

- Bestimmte Werturteilen bezüglich Prostitution oder Migration sowie Täter- bzw. Opferklischees bei den Beamten/Beamtinnen erzeugten bei den Frauen Gefühle, nicht ernst genommen, herabwürdigend behandelt, diskriminiert oder als nicht glaubwürdig eingeschätzt zu werden.

- Wurden Frauen ausschließlich als Migrantinnen ohne Aufenthaltserlaubnis angesehen und ausgewiesen oder ausschließlich als Betroffene von häuslicher Gewalt betrachtet konnten sie nicht als Opfer von Menschenhandel identifiziert werden.

Zum Teil hatten die Frauen ein unklares, verunsicherndes Bild über die Strafbarkeit ihrer Handlungen im Umfeld des Menschenhandels wie z.B. das Führen einer Legalisierungsehe, Steuerhinterziehung, Ausübung von Prostitution. Ihnen fehlten verlässliche Informationen über ihren Legalstatus. Im Kontakt mit der Polizei wurden vor allem Nachteile antizipiert. Angst vor der Polizei entstand teilweise aufgrund von entsprechenden Drohungen der Täter/innen, teilweise aufgrund eigener Erfahrungen im Herkunftsland oder in Deutschland. Mangelnde Rechtssicherheit sowie das Bild der Polizei als sanktionierende Institution waren starke Motive gegen eine Aussage bei der Polizei.

Aus der Sicht der Frauen erzeugte die Mitteilung einer drohenden Haft oder eine Inhaftierung einen höchstmöglichen Druck. Dieser konnte in einigen Fällen eine Aussage erzwingen, in anderen jedoch nicht, z.B. wenn die Täter als stärker als die Polizei oder die Polizei als korrupt eingeschätzt wurden. Eine Wahrnehmung der Polizei als unterstützend wurde unmöglich, wenn Frauen als (Mit-) Täterinnen die Doppelrolle der Frauen als Tatverdächtige und Betroffene nicht schnellstmöglich aufgelöst wurde.
Unabhängig von der Art der polizeilichen Intervention erhöhte sich die Kooperationsbereitschaft der Frauen, wenn sie in der Lage waren, die Polizei als eine schützende oder unterstützende Einrichtung wahrzunehmen. Dies brauchte Zeit, Unterstützung und eine Vorgehensweise, bei der Angst nicht verstärkt und antizipierte Nachteile ausgeräumt wurden, was am ehesten durch eine Vermittlung an Fachberatungsstellen gelang.

Ausweiskontrollen in Prostitutionsbetrieben konnten kurzfristig dazu dienen, Frauen ohne rechtmäßigen Aufenthaltsstatus räumlich aus dem Umfeld der Täter/innen zu lösen und zu befragen - eine Voraussetzung dafür, dass sie sich überhaupt äußern konnten - aber gleichzeitig adressierte diese Maßnahme die Betroffenen von Menschenhandel als Beschuldigte im Sinne eines Verstoßes gegen das Ausländergesetz und bestätigte somit vorherrschende Ängste vor der Polizei. Mangelnde Rechtssicherheit sowie das Bild der Polizei als sanktionierende Einrichtung waren starke Motive gegen eine Aussage bei der Polizei.

\section{Konstruktionen des Tätersystems und der Polizei}

Determinanten sind für Betroffene in ihrer subjektiven Deutung wesentlich, selbst bei einem ähnlichen Ausmaß und ähnlichen Formen von Gewalt macht es einen Unterschied, ob ihre Deutung der Situation die Möglichkeit eines Entkommens einschließt oder nur ein auswegsloses Ausgeliefertsein. Den Deutungen der Gewalt liegen reale Erfahrungen zugrunde, doch sind die Deutungen zugleich auch immer Bewältigung dieser Erfahrungen, denen im Kontext der eigenen Geschichte ein bestimmter Sinn verliehen wird. Bezogen auf das Tätersystem und die Polizei ließen sich mehr oder weniger ausgeprägte implizite Deutungen und subjektive Theorien rekonstruieren.

\section{Implizite Theorien über die Polizei}

Die impliziten Theorien über die Polizei speisten sich aus drei Quellen: aus eigenen für Herkunftsländer spezifischen Erfahrungen, aus einem kursierenden Allgemeinwissen, Erzählungen und Gerüchten unter Kolleginnen und aus von Tätern vermittelten Konstruktionen.

Explizite Theorien über die Polizei im Herkunftsland waren negativ, wenn sie vorkamen - eine positive Theorie dieser Polizei kiam nicht vor - und schlossen an einen 
Diskurs über Armut, Unterdrückung und Ungerechtigkeit an: "If you are a rich man (...) and hit a poor man and when I go and report it to the police (...) you just give them money, and they will instead put me in jail" oder "In Weißrussland arbeitet ja die Polizei nur für die, die zablen. Und die Kriminellen zablen an die Polizei dort, auch die Zubälter" oder "Die Polizei kann man kaufen". Aus der Perspektive der Frauen selbst bedeutete dies, dass keine Hilfe zu erwarten war.

Die Annahmen über die Polizei in Deutschland waren ebenfalls im Einzelnen sehr unterschiedlich. Die Konstruktion der Polizei als sanktionsmächtiger Instanz betrifft vor allem Frauen mit einem nicht rechtmäßigen Aufenthalt. Die Angst vor der Polizei wurde von Tätern gezielt geschürt, die warnten, die Polizei werde die Frauen abschieben, bestrafen, ins Gefängnis werfen, bzw. die entsprechende Informationen z.B. zur Rechtmäßigkeit von Prostitution vorenthielten: „Zum Beispiel ich, ich habe mein ganzes Leben vorher in Angst gelebt, auch was du hörst von deinem Chef, der sagt immer, die Polizei, die böse, du darfst nicht mit der Polizei zusammen arbeiten ", Darstellung der Polizei als „schlimmster Feind“ oder „Die haben immer gesagt, ja wenn du zur Polizei gehst, dann bringt dir das eh nichts, weil ((weint)) die Behörden glauben eine Deutschen, also deutschen Bürger eher als irgendeine osteuropäischen Hure".

Ebenfalls über die Täter vermittelt, aber durchaus an Theorien über die Polizei aus dem Herkunftsland anschließend, ist das Bild der Zusammenarbeit von Polizei mit den Tätern. Die Täter würden von der Polizei im Vorfeld von Durchsuchungsmaßnahmen in den Betrieben informiert (insbesondere bei bandenmäßig organisierter Kriminalität als Prostitutionskontext), Polizisten seien Kunden oder „ein Freund des Hauses “ und würden ,von uns und von den Drogen" abbekommen. „Bei uns war Polizist und er hat gesagt, also dass meine Chef ist böse oder noch dass ich muss anzeigen (...) Ich habe sofort gesagt, nein, denn ich habe ganz genau gewusst, dass er hat Bekannte von Polizei. Er immer informiert, wann kommt die Polizei. "Diese Konstruktion wurde nicht nur als Konstruktion der Täter zitiert, sondern eigene Erfahrungen der Frauen bescheinigten ihr Gültigkeit.

\section{Konstruktionen der Tätermacht}

Gewalt und Drohung mit Gewalt konstituierten im Verlauf der Erzählung die Macht der Täter bzw. des Tätersystems und be- gleiteten die Durchsetzung von Täterforderungen, das Brechen von Widerstand, Einschüchterung und das Aufgeben von Fluchtgedanken („Deshalb habe ich da auch so lange ausgehalten“; „Ich wurde gezwungen zu arbeiten. Und dann hatte ich keine Möglichkeit mehr, damit aufzuhören"). Die subjektive Bedeutung der Drohungen und Gewalt lag darin, dass sie als durchgehende (,immer“, „immer wieder“) Merkmale der Praxis von Tätern als Personen (wenn z.B. der Ausbeuter der Ehemann war: häusliche Gewalt) oder Tätersystemen (bei bandenmäßiger Organisation: hochgradig ritualisierte Gewaltabläufe) verallgemeinert wurden. Gewalt ist ein wesentliches Merkmal für die subjektive Wahrnehmung des Täters/der Täterin oder Tätersystems als übermächtig und gefährlich, während die eigene Handlungsfähigkeit verloren geht.

Die Täterstrategie des Beeindruckens, Versprechens und Verliebtmachens und die Einstellung zu Prostitution als Arbeit über einen ersten Zeitraum hinweg konnte eine Deutung der Beziehung zwischen Opfer und Täter unterstützen, dass die Frau ihre Ziele, z.B. Geld zu verdienen, an der Seite des Täters verwirklichen könne. Die Polizei war dann lästiger bis gegnerischer Außenstehender, der die Möglichkeit des Geldverdienens untergräbt. Dies war auch dann so, wenn das Opfer des Menschenhandels in die Prostitution einwilligte und dabei der Fiktion folgte, es handele sich um eine Geschäftsbeziehung zu beiderseitigem Nutzen. Diese Deutung wurde dann aufgegeben, wenn mit Gewalt die Arbeit in der Prostitution erzwungen oder die Ausbeutung zugespitzt wurde.

Wenn berichtet wurde, dass die Täter über Aktionen der Polizei z.B. Razzien vorab informiert gewesen seien, dann wurde in der Beziehung zwischen Täter und Polizei den Tätern ebenfalls Überlegenheit und eine Macht zugeschrieben, die bis in die Organisationsstrukturen der Polizei hineinreichte. Bei einigen Befragten war das Tätersystem als so mächtig konstruiert, dass die Polizei sie niemals schützen könnte (,Man musste aussagen für den Zeugenschutz, dafür war ich nicht bereit, weil so viel können sie gar nicht schützen").

\section{Druck von allen Seiten bietet Ansatzpunkte für Intervention}

Die Bedeutung der Determinanten konnte sich faktisch und subjektiv verändern, z.B. aufgrund von Gewalteskalationen, über $\mathrm{Zu}$ gang zu Informationen durch Beratung, über die Vermittlung von Schutz oder Aufenthalt durch die Polizei. Ihre Wirksamkeit hing ab z.B. von der aktuellen Situationseinschätzung der Frau bzw. dem Prozess der Lösung vom Täter und somit von dem Zeitpunkt, an dem der Polizeikontakt stattfand. Daher wurden die Wirkungen der Determinanten im Rahmen der Analyse eingebettet in komplexere Prozesse und in Bezug zu polizeilichem Handeln und Beratung gesetzt und in ihrer Wechselwirkung untersucht:

- Druck, der von Täter/innen ausgeübt wird (z.B. Gewalt, Bedrohung, 'Schuldenfalle', Ankündigung von Ausweisung im Fall von Polizeikontakt).

- Druck, der von polizeilichen Maßnahmen ausgeht (z. B. Ankündigung von Haft oder Ausweisung).

- Druck, eigene Migrationsziele zu erreichen (z.B. eigene Existenzsicherung, Versorgung von Angehörigen im Herkunftsland, Verbesserung der Zukunftsperspektive).

Auf die Interviewpartnerinnen ohne rechtmäßigen Aufenthaltsstatus wirkte in der Regel Druck von allen drei Seiten. Insbesondere bei Frauen dieser Gruppe, die von der Polizei aufgegriffen wurden und zunächst nicht zu einer Aussage bereit waren, ließ sich daher die Entwicklung ihrer Aussagebereitschaft im Rahmen verschiedener Konstellationen von Druck aufzeigen. Es konnten dabei Ansatzpunkte systematisiert werden, wie die Polizei eine Bereitschaft der Interviewpartnerinnen zur Aussage dadurch unterstützen konnte, dass sie an dem Druck, der jeweils vorhanden war, ansetzte, soweit der rechtliche Spielraum gegeben war.

- Druck der Frauen, ihr Migrationsziel $\mathrm{zu}$ erreichen, nutzen: Optimierung des Angebotes an die potentiellen Opferzeuginnen mit Hinblick auf die Eigeninteressen der Frauen (Schutz, Aufenthalt, Zugang zu Bildung und zum Arbeitsmarkt);

- Druck vonseiten der Täter/innen mindern: Täterdrohungen im Fall einer Aussage entkräften über verlässliche Informationen, Sprachmittlung, Verhaftung der Täter/innen, Schutz, Entlastung der Frauen von der alleinigen Verantwortlichkeit, die Täter/innen zu belasten),

- Druck der Polizei „regulieren“: durch z.B. schnelles Auflösen der Doppelrolle als Straftäterin und Opfer, Vermeidung von Haft, schnelle Vermittlung in Beratung. Wo Druck seitens der Polizei (z.B. eine Inhaftierung) den gesetzlichen Vor- 
gaben entsprechend vollzogen werden musste, kann eine gute Kooperation mit der Justiz, das schnelle Einschalten einer Fachberatungsstelle und Rechtsberatung dennoch das subjektive Empfinden der Frauen positiv beeinflussen.

Die erfolgreichen Verläufe zeigten, dass gleichzeitig alle Ansatzmöglichkeiten genutzt werden mussten.

\section{Viktimisierungsverläufe und Opferdeklaration}

Über die biografischen Vorsaussetzungen und Prozesse, die einer Aussage im Falle von Menschenhandel vorgelagert sind, ist wenig bekannt. Die Wahrnehmung (Selbst- oder Fremdwahrnehmung), Opfer zu sein, ist eine erste, notwendige, aber nicht hinreichende Bedingung für die Offenlegung des Opferstatus gegenüber Polizei oder Dritten. Diese und auch die weiteren Schritte zu einer Aussagebereitschaft und von dort zu einer Zeuginnenaussage erfolgen nicht zwangsläufig. Auch wenn dies nahe liegend erscheint, so folgt doch keineswegs immer auf die Ausbeutung in einem direkten zeitlichen Zusammenhang und ohne weitere Anstöße die Opferwahrnehmung und Opferwahrnehmung und Opferdeklaration anderen gegenüber fallen nicht immer zusammen. Wesentliche Stufen in dem theoretischen Modell (Baurmann 1983:25) sind:

- sich als Opfer wahrzunehmen,

- anderen den Opferstatus mitzuteilen,

- sich gegen die Täter/innen zu stellen,

- eine Bereitschaft zur Aussage zu entwickeln,

- auszusagen und

- bei der Aussage zu bleiben.

Jeder dieser Stufen kann ein Wendepunkt vorausgehen oder ein Wendepunkt führt dazu, dass diese Stufe nicht erreicht wird und eine Aussagebereitschaft sich verzögert oder nicht zustande kommt. Die Opferdeklaration konnte sowohl von der Betroffenen selbst gegenüber der Polizei oder Dritten erfolgen, als auch unabhängig von der Selbstdeklaration als Fremddeklaration durch die Polizei oder Dritte vorgenommen werden. Eine Selbstdeklaration setzte eine Selbstwahrnehmung als Opfer voraus, die Opferwahrnehmung kam aber auch ohne Umsetzung in eine Opferdeklaration vor, wenn Barrieren der Offenbarung nicht überwunden werden konnten. Es kam auch der Fall vor, dass eine Opferdeklaration durch die Betroffene aus strategischen Gründen,
z.B. um eine Inhaftierung oder Ausweisung abzuwenden, erfolgte, ohne dass die Frau sich selbst als Opfer empfand, z.B. weil sie ihre Situation als akzeptabel erlebte.

Der wahrgenommene oder deklarierte Opferstatus konnte sich auf unterschiedlich Adressaten beziehen: Die Interviewpartnerinnen sahen sich nicht nur als Opfer der Täter/innen, sondern auch der Polizei bzw. anderer Institutionen. Bezogen auf Täter/ innen und Arbeitsbedingungen konnte der Opferstatus inhaltlich gefüllt sein als: Opfer des Zwangs zur Prostitution gegen den eigenen Willen; Opfer von Gewalt, Einschüchterung und Bedrohung der eigenen Person oder Angehöriger; Opfer von Täuschungen und falschen Versprechungen; Opfer von Betrug und Ausbeutung wie vorenthaltenem Verdienst oder willkürlich festgelegten Schulden; Opfer von inakzeptablen und belastenden Arbeitsbedingungen, auch wenn Frauen bereit waren, in der Prostitution zu arbeiten.

Für die Auswertung wurden fallbezogen Verlaufskurven erstellt. Basis für die Rekonstruktion der Binnengliederung der Verläufe waren zum einen inhaltlich berichtete Zäsuren (,,und dann habe ich beschlossen...", „plötzlich dachte ich: das geht nicht so weiter...", „da habe ich das erste Mal..." etc.), zum anderen ein Wechsel in der sprachlichen Darstellungsform, der eine Veränderung in der subjektiven Handlungsmächtigkeit anzeigt, wie z.B. ein Wechsel von einer vor allem aktiven Form ("Ich habe getan / beschlossen / gezeigt / gearbeitet" etc.) oder der Form des Wollens und Durchsetzens hin zu erleidenden Formen (,sie haben mich gebracht / geschlagen" etc.), Formen des Zwangs („ich musste...", „sie haben mich gezwungen...") oder der Hilf- und Ausweglosigkeit und Ineffektivität ("ich habe versucht, aber...", „immer wieder wollte ich, aber...", „ich konnte nicht..." etc.). Der Wechsel in dem dominierenden sprachlichen Modus im Interview entspricht Zäsuren der Rückgewinnung oder des Verlustes von Handlungsmächtigkeit. Die Abwesenheit von eigener Handlungsmächtigkeit - entweder explizit benannt oder indirekt festgemacht an dem sprachlichen Agencymodus des Erleidens - wurde als Aspekt der subjektiven Opferwahrnehmung definiert. ${ }^{4}$

In der Forschung zu Opfern von Straftaten oder Menschenhandel gibt es wenig direkte Anknüpfungspunkte für eine theoretische Konzipierung der biografischen Prozesse der Viktimisierung. Es wurde auf die Erkenntnisse der Bewältigungsforschung, des Co- ping (Lazarus/Launier 1978) zurückgegriffen, die helfen, Viktimisierungsprozesse zu verstehen. Ein spezieller Ansatz des Coping ist die Veränderung kognitiver Strategien, d.h das Denken über die den Stress auslösenden, bedrohlichen Faktoren wird verändert, sie werden aus einem anderen Blickwinkel betrachtet und es kommt zu einer Neubewertung der Situation. Eine besondere Rolle kommt hier dem zu, was über die Bewältigung von Traumatisierungen bekannt ist. ${ }^{5}$ Traumaforschung hat unterschiedliche Modelle von Trauma entwickelt (Typ I und Typ II) und zeigt, dass die erforderlichen Bewältigungsstrategien prozesshaft und kontextabhängig und ein komplexes $\mathrm{Zu}$ sammenspiel von Individuum und Umwelt sind (vgl. Verlaufsmodell psychischer Traumatisierung von Fischer/Riedesser 1999). Zum Verständnis des Erlebens erzwungener Prostitution gehört das Verständnis des Vergewaltigungsstraumas (Kretschmann 1993:43). Alle diese Ansätze können nur Anregungen bieten, denn die Situation von Betroffenen von Menschenhandel ist von vielen Spezifika gekennzeichnet und zudem sehr heterogen.

\section{Muster von Viktimisierungsverläufen}

Die hier vorgestellte Studie konnte drei hauptsächliche und zwei weitere Muster von Viktimisierungsverläufen identifizieent und die Dynamik mit Wendepunkten und zu überwindenden Barrieren beschreiben.

\section{Muster 1: Durchgehende Opferwahrnehmung - Bewältigung durch Aufrechterhalten des Fluchtgedankens}

Merkmal dieses Modells des Viktimisierungsprozesses ist eine Opferwahrnehmung von Anfang an, d.h. ab dem Moment, in dem den Frauen gesagt wurde, dass sie in der Prostitution zu arbeiten hätten bzw. als ihnen klar wurde, in welcher Situation sie sich befanden. Alle waren über die Art der Arbeit, die sie erwartete, getäuscht worden, und alle lehnten die Prostitution strikt ab. Die Selbstwahrnehmung als Opfer blieb durchgehend erhalten. Alle erlebten Gewalt und/oder Zwang.

Es gab hinsichtlich der Opferwahrnehmung, die im Rahmen der Bewältigung von Gewaltsituationen Phasen der Latenz durchlaufen konnte, keine Wendepunkte. Aussagebereitschaft konnte von Anfang an bestehen, es konnten Barrieren davor liegen und/oder Wendepunkte zu einer Aussage- 
bereitschaft führen, wie z. B. eine Eskalation der Gewalt oder eine Intervention. Zur Polizei und Aussage gelangten diese Frauen auf allen möglichen Wegen, von der selbst initiierten Flucht direkt zur Polizei, über die Vermittlung Dritter oder von Beratungsstellen bis zum völligen Zufall.

Das Zwangsverhältnis war bei diesem Muster von eher kürzerer Dauer -weniger als 12 Monate, meist sehr viel kürzer, auch wenn zwei oder drei Monate sich wie „zwanzig oder dreißig Jahre anfühlten “. Teilweise entschlossen sich die Frauen sofort zur Flucht und setzten diesen Entschluss bei der ersten Gelegenheit um (,,Von Anfang an habe ich gewusst, dass ich abhauen werde, da ich dort nicht leben wollte (...) Ich habe nur auf eine Möglichkeit gewartet, auf den Moment, in dem sie vergessen, die Tür oder das Auto $z u$ schließen. ") oder sie mussten einige zeit aushalten, verloren aber die Fluchtperspektive nicht aus den Augen, selbst wenn ihre Handlungsfähigkeit zeitweilig beeinträchtigt ist („Ich wollte immer weglaufen, sie haben mit ständig Angst eingejagt, irgendwann gibt man auf").

\section{Muster 2: Aufgeschobene Opferwahrnehmung - Arrangement und Empörung als Teil der Bewältigung}

Zwei weitere Muster von Viktimisierungsprozessen sind dadurch gekennzeichnet, dass eine Opferwahrnehmung erst verzögert relevant wird. Einmal tritt eine anfangs vorhandene Opferwahrnehmung im Zusammenhang mit der erzwungenen Prostitution im weiteren Verlauf wieder zurück, um zu einem späteren Zeitpunkt aktualisiert zu werden. Das andere Mal ist zunächst keine Opferwahrnehmung vorhanden und sie entwickelt sich erst im weiteren Fallverlauf. Durch die erst spät zum Tragen kommende Opferwahrnehmung unterscheiden sich diese Verläufe von dem obigen Muster der durchgehenden Opferwahrnehmung.

Eine Opferwahrnehmung von Beginn an kann unter den Bedingungen von Druck, Kontrolle, Isolation, Bedrohung und/oder aussichtslosen Situationen und der Unmöglichkeit einer Flucht oder Rückkehr zurücktreten und phasenweise ganz verschwinden. Anders als beim ersten Verlaufsmuster verändert das Arrangement die Perspektive. Frauen, aus deren Verläufen das Muster konstruiert wurde, sprachen von der Prostitution als ihrer "Arbeit" und nicht mehr als Zwang, von „Einarbeitung“, und davon, wie sie die Regeln des Metiers erlernten
„Also fing ich an zu arbeiten. Sie zeigte mir, wie ich die Arbeit zu machen hatte, denn ich hatte das vorher noch nie gemacht. Sie erklärte mir alles, was ich wissen musste." Sie sagten, dass ihnen nichts anderes übrig blieb und ein Kooperieren mit den Täter/innen Gewalt und Risiken zu verringern versprach. Durch dieses Sich Einfinden in die Situation wurden Freiräume möglich (,Am Anfang kannte ich mich ja noch gar nicht aus... und dann bin ich auch rausgegangen ohne Papiere, weil sie das auch gemacht haben, und kannte mich besser aus"; „Wir durften raus"). Es handelte sich um Frauen aus Afrika und Asien, die mit der Rückzahlung hoher Reisekosten konfrontiert wurden und für die eine selbst initiierte Rückreise unmöglich war.

Kennzeichen dieses Musters sind sehr lange, meist mehrere Jahre dauernde Verläufe, teilweise wurden während dieser Zeit Kinder geboren. Es gab psychischen Druck aber keine sadistische Gewalt.

Der Verlauf in diesem Muster kann auch so aussehen, dass von Beginn an ein Einverständnis mit der Tätigkeit in der Prostitution und keine Opferwahrnehmung vorliegt. Prostitution wird mit einer professionellen Haltung als Arbeit angesehen und ohne Ekelgefühle und Widerstand ausgeübt. Die Situation wird als vertraglich festgelegtes Geschäft und als eine Erwerbsmöglichkeit zum Erreichen der Migrationsziele gedeutet. Ein Wendepunkt als Herausbildung einer subjektiven Opferwahrnehmung wird bei diesem Verlauf nach einiger Zeit nicht über die Prostitution als solche, sondern über die Rahmenbedingungen ausgelöst, die als ungerecht empfunden werden und Empörung auslösen. Dies können z.B. inakzeptabel definierte Arbeitsbedingungen, Vorenthalten des Verdienstes, einschränkende Kontrollen der Täter/innen oder Gewalt sein. („Ich musste jeden Tag arbeiten, ob ich meine Periode hatte oder nicht. "). Dritte ebneten den Weg zur Polizei oder zur Beratungsstelle.

\section{Muster 3: Biografisch durchgehende Opferwahrnehmung: Bewältigung durch die Vermeidung einer Konfrontation mit den Täter/innen}

Merkmal dieses Musters des Viktimisierungsprozesses ist eine schon biografisch früh und weit vor dem Menschenhandel einsetzende Opferwahrnehmung. Die zugehörigen Fälle, aus denen das Muster gebildet wurde, sind Biografien die durchgehend mit Gewalt belastet waren: Misshandlung - auch sexuelle Gewalt - in Kindheit und
Jugend, Vernachlässigung und Verlassenwerden durch die Eltern, Aufwachsen unter schlechten Bedingungen im Heim oder auf der Straße, Alkoholismus der Eltern, auch Tod der Eltern, desolate Lebensverhältnisse, keine ausreichende Versorgung, Gewalt in Beziehungen, teilweise sehr früher Beginn der Prostitution in der Kindheit. Es gab - vor allem schlechte - Erfahrungen mit Anzeige und Gerichtsverhandlung wegen früher Gewalt, Versuche, sich aus schädigenden Familienverhältnissen zu lösen, sich allein durchs Leben zu schlagen. Die Frauen erlebten ihre Situation weniger als Unrecht sondern eher als Unglück bzw. Schicksal.

Die Dynamik des Musters ist gespeist vor allem davon, dass die chronische Gewalt generell Überlebens- und Bewältigungsstrategien erfordert und dass die Bewältigung des Menschenhandels eingebettet ist in die individuelle biographische Bewältigung einer Vielzahl von meist traumatisierenden Erlebnissen, die durch jeweils neue Gewalt oder Drohung wieder aktiviert werden. Ein Beispiel einer Frau aus Tschechien: Als Kind lief sie aus einer desolaten Familie weg und kam als Straßenkind in die Prostitution. Von der Polizei wurde sie in ein Kinderheim gebracht, an das sie schreckliche Erinnerungen hat. Später geriet sie wieder an die Täter/innen, die sie weiter verkauften. Es gab keine Wendepunkte bezogen auf ihre Opferwahrnehmung und Aussagebereitschaft, aber hohe Barrieren der Angst vor den Tätern/innen, die ihre kleine Tochter in ihrer Gewalt hatten. Selbst in der Haft, die sie als massive Retraumatisierung erlebte, sagte sie deshalb nicht aus. Mit Hilfe von Kolleginnen wandte sie sich an eine Beratungsstelle. „Ich war kaputt von Männer, von die Arbeit, von viele Gedanken, von den Angst um die Kleine." Der Wendepunkt hin zur Aussagebereitschaft war erst möglich, als der Schutz der Tochter durch die Beratungsstelle gewährleistet wurde.

\section{Muster 4: Abwesende Opferwahrnehmung: Bewältigung durch Umdefinieren der Situation}

Merkmal dieses Modells des Viktimisierungsprozesses ist die am Anfang fehlende Wahrnehmung als Opfer von Menschenhandel, die sich aber, anders als bei dem Muster 2 „Empörung“ auch im Verlauf der Zeit nicht herausbildet. Die Arbeit in der Prostitution ist geplant und akzeptiert als Möglichkeit, Geld zu verdienen und das Migrationsziel zu erreichen. Die Interview- 
partnerinnen konnten sich z.B. als Opfer deutscher Behörden sehen.

$\mathrm{Zu}$ einer Aussagebereitschaft kam es mit einer strategischen Übernahme einer externen Opferdeklaration, um z.B. eine Haftentlassung zu erwirken oder um den Aufenthalt und die Migrationsziele zu sichern. Ein charakteristisches Beispiel ist eine Interviewpartnerin aus Rumänien. Sie ging freiwillig in die Prostitution und verdiente ausreichend, um ihr Migrationsziel zu erreichen. Sie war mit den Arbeitsbedingungen vorerst zufrieden und nahm durchgehend keine Opferdeklaration im Kontext des Menschenhandels vor, es gab hier keinen Wendepunkt. Sie erklärte sich mit der Form der Ausbeutung - sie gab die Hälfte ihres Verdienstes ab - einverstanden. Ein Wendepunkt zur Aussagebereitschaft trat ein, um Nachteile zu vermeiden. Sie wurde inhaftiert wegen des Verdachts der Mittäterschaft und der Behinderung der Ermittlungen und eine Haftentlassung wurde über die Aussage möglich.

Anknüpfungspunkte für polizeiliches Handeln konnten wie folgt identifiziert werden:

- Bei den Verlaufsmustern 1, 3 und 4 geht es vor darum, die Opferwahrnehmung (wieder) zu fördern. Dies kann durch verlässliche rechtliche Information, eine Reduzierung der Angst vor der Polizei durch respektvolles und fürsorgliches Verhalten vor, während und nach der Vernehmung und vor allem durch die Vermittlung an eine Fachberatungsstelle erfolgen.

- Bei den Verlaufsmustern 2 bis 5 ist eine Intervention erforderlich, die geeignet ist eine Opferdeklaration und - als spezielle Form der Opferdeklaration - eine Aussagebereitschaft zu bewirken. Auch hier spielt verlässliche Information eine wichtige Rolle, aber auch ein Ernstnehmen der individuellen Migrationsziele.

\section{Bedeutung von Beratung für die Aussagebereitschaft}

Beratung war eine Determinante von großer Bedeutung in mehrfacher Hinsicht. Wenn der Kontakt zur Fachberatungsstelle vor einer Aussage bei der Polizei zustande kam, ergab sich die Chance zur Förderung der Aussagebereitschaft, insbesondere zur Vermittlung von Information, Klärung der Perspektive und Motivation. Nach einer ersten Aussage bei der Polizei behielt der Kontakt zur Beratung in allen Fällen große Bedeutung für das weitere Verfahren, z.B. in dem die Zeugin in die Lage versetzt wurde, sich klar gegen die Täter/innen zu stellen, nichts zurückzuhalten, ihre Aussage auszuweiten und ihre Angst zu überwinden sowie für das Aufrechterhalten der Aussagebereitschaft während der Wartezeit auf ein Gerichtsverfahren und während des Verfahrens.

Beratungs- und Unterstützungsangebote waren den meisten Interviewpartnerinnen nicht bekannt, bevor sie dorthin vermittelt wurden. Sie waren darauf angewiesen, dass andere ihnen den Zugang ermöglichten, indem sie ihnen die Information gaben oder den Kontakt für sie herstellten. Dieser Kontakt zur Beratung wurde in der Mehrheit der Fälle durch die Polizei erst nach einer Vernehmung und Aussage hergestellt, was den Zeuginnen die Chance nahm, sich gut informiert für oder gegen eine Aussage zu entscheiden.

Die inhaltsanalytische Querauswertung zeigt, welche Aspekte von Beratung eine Gegenwelt zu bisherigen Erfahrungen darstellen, die den Interviewpartnerinnen neue Handlungsoptionen und Entscheidungsmöglichkeiten eröffnen. Zentral waren Vertrauen und Schutz („Das wichtigste war, dass sie mir meine Angst genommen hat." "Sie hat mich überzeugt, dass ich keine Angst haben muss, sondern kämpfen. Ich brauche keine Angst zu haben vor der Gerichtsverhandlung, ihn in seine Augen sehen. Ich muss kommen und sagen, was hast du gemacht? Kämpfen um die Kinder, um mich selbst."), Kommunikation und Verständigung (, Und die haben Dolmetscherin und die Frauen sollen nicht Angst haben, dass keiner versteht und dass sie sind nachher ausgelacht."), ein sicherer Ort (,Da war es irgendwie anders, da war es so schön, ich habe mich gefühlt wie zuhause."), die Sicherung der aktuellen Existenz („Ich habe so viel bekommen, ich hätte noch nie im Leben mir das träumen können.") und Selbstbestimmung (,Es wurde immer mit mir abgesprochen, also es wurde nichts hinter meinem Rücken gemacht und ich wurde über jeden Schritt informiert.“). Zusammen ergibt dies ein Konzept des Empowerment.

\section{Schlussfolgerungen}

Die Interviews erheben die individuelle Perspektive der Betroffenen und bieten Einblick in ihre Entscheidungsprozesse. Je nachdem, welche Aspekte der Zwangssituation für die Frauen den Ausschlag gaben konnten Muster von Viktimisierungsprozessen herausgearbeitet werden, die Ansatzpunkte für Intervention sichtbar machen.
$\mathrm{Zu}$ den Voraussetzungen eine Aussagebereitschaft zu fördern oder zu initiieren gehört das Erkennen, in welcher Phase von welchem Muster des Viktimisierungsprozesses sich die Frau befindet, das Erkennen der Barrieren, ein auf das Verlaufsmuster abgestimmtes, gezieltes Vorgehen, die Aussagebarrieren zu überwinden, sowie eine aufmerksame Verhinderung des Aufrichtens neuer Barrieren. Die sensiblen Momente oder „Zeitfenster“ zu erkennen, in denen eine Opferwahrnehmung manifest werden und zu einer Opferdeklaration, Aussagebereitschaft und letztendlich einer Aussage führen kann, ist Voraussetzung für gelingende Intervention.

Die räumliche Trennung und Herauslösung der Opfer aus dem Tätersystem ist von großer Bedeutung, weil erst sie Erfahrungen und Deutungen ermöglicht, die die behauptete Macht der Täter/innen und die Abhängigkeit von ihnen verändern können.

Die subjektiven Darstellungen von Vernehmung als persönlichem Polizeikontakt zeigen die Möglichkeit einer solchen Veränderung. Der Einschnitt wird durch den Wechsel der Thematisierungsebene angezeigt: Die Polizei ist nicht mehr der abstrakte ,Feind', sondern die Vernehmung wird als Begegnung von Menschen erzählt. Wie die Vernehmungen im Interview beschrieben und bewertet werden, zeigt eine hohe Sensibilität der Betroffenen, ob die Polizei das Opfer zurückverweist in das Tätersystem (indem sie z.B. nicht als Opfer erkannt werden, als „Nutte“ angesprochen und beleidigt werden, oder ihnen mit strafrechtlicher Sanktionierung von Passvergehen gedroht bzw. sie als Mittäterin angesehen werden, was mit einem Opferstatus nicht vereinbar ist) oder eine vertrauensvolle, fürsorgliche Situation schafft. Der Wunsch der Frauen nach Respekt und Schutz lässt sich als Voraussetzung für eine Veränderung interpretieren: Der Wechsel aus dem Tätersystem in die Kooperation der Polizei bedeutet eine bislang nicht vorgesehene und neue Beziehung zwischen Opfer und Polizeibeamt/in, die der Absicherung bedarf.

Wenn die Polizei am Zuge ist, so lassen sich die Ergebnisse weiter interpretieren, kann sie das Deutungsmuster der Beziehungen zwischen Täter/innen, Polizei und Opfer dekonstruieren (vorausgesetzt sie erkennt das Opfer als Opfer). Die Entmachtung der Täter, eine Stärkung der Betroffenen durch Information, ein Aufzeigen von Perspektiven, die Vermittlung an und Kooperation mit Fachberatungsstellen und effektiver Schutz 
widerlegen die Konstruktion der Macht der Täter/innen und der Verfolgerrolle der Polizei. Letztlich wird mit einer Aussage verlangt, dass die Betroffene den Täter/innen schadet und deren Strafverfolgung unterstützt. Dies bedeutet ein hohes Risiko, eine große Belastung und eine Umkehrung der bisherigen Deutung, die ohne den Schutz der Polizei nicht möglich ist. Die subjektive Bedeutung der Gestaltung der Vernehmung ließe sich so auch interpretieren als das Nutzen der einzigen ,Macht', die das Opfer hat: die Macht, die Aussage zu verweigern oder auszusagen.

\section{Literatur}

Balß, Rudolf; Baurmann, Michael C.; Lieser, Udo; Rein, Dieter; Voß, Hans-Georg W. (2001) Opfer und Zeugen bei der Polizei, Polizei + Forschung Bd. 11, BKA (Hrsg.)

Baurmann Michael C; Schädler Wolfram (1999) Das Opfer nach der Straftat - seine Erwartungen und Perspektiven. Eine Befragung von Betroffenen zu Opferschutz und Opferunterstützung sowie ein Bericht über vergleichbare Untersuchungen. BKA Forschungsreihe 22 2. Auflage. BKA, Wiesbaden

Fischer G and Riedesser P (1999) Lehrbuch der Psychotraumatologie, 2. Auflage. Reinhardt, München

Gallagher, Anne; Paul Holmes (2008) Developing an Effective Criminal Justice Response to Human Trafficking. Lessons From the Front
Line, International Criminal Justice Review, Volume 18 Number 3, 318-343

Goodey, Jo (2008) Human trafficking: Sketchy data and policy responses, Criminology \& Criminal Justice, Vol: 8(4): 421-442

Helfferich, Cornelia; Kavemann, Barbara; Rabe, Heike (2010) Determinanten der Aussagebereitschaft von Opfern des Menschenhandels zum Zwecke sexueller Ausbeutung. Eine Opferbefragung, BKA (Hg.), Köln, Luchterhand

Helfferich Cornelia (2005) Die Wahrnehmung der eigenen Handlungsmacht und die Konstellation Opfer - Polizei - Täter bei häuslicher Gewalt. Die subjektive Perspektive von Frauen. In: Kury Helmut; Obergfell-Fuchs Joachim (Hg.) Gewalt in der Familie. Für und Wider den Platzverweis. Lambertus, Freiburg

Helfferich, Cornelia (2005): Qualität qualitativer Daten. Ein Schulungsmanual zur Durchführung qualitativer Einzelinterviews. Leverkusen: Verlag für Sozialwissenschaften. 2. Auflage

Herz Annette; Minthe Eric (2005) Straftatbestand Menschenhandel. Verfahrenszahlen und Determinanten der Strafverfolgung BKA Forschungsreihe 31. Luchterhand, München

Kretschmann Ulrike (1993) Das Vergewaltigungstrauma. Westfälisches Dampfboot, Münster

Reemtsma Jan Philipp (ohne Jahr) Was sind eigentlich Opferinteressen? Ansprache1 zur Feier des 25. Jahrestag der Gründung des „Weißen Ring “, Hamburg

Schädler, Wolfram (2002) Die Opfer von Menschenhandel. Viktimologische Perspektiven für Risiken und Chancen der Strafverfolgung im Bereich der organisierten Kriminalität. In: Agisra Rundbrief Nr. 32/33, S. 22-26
Sieber Ulrich; Bögel Marion (1993) Logistik der Organisierten Kriminalität. BKA, Wiesbaden

van Duyne Petrus C; von Lampe Klaus; Passas Nikos (Hg.) (2000) Upperworld and Underworld in Cross-Border Crime. Wolf Legal Publishers, Nijmegen

Voß Hans-Georg W (2001) Professioneller Umgang der Polizei mit Opfern und Zeugen. Eine Evaluationsstudie. BKA Forschungsreihe 12. Luchterhand, München

\section{Fußnoten}

1 Die Studie wurde 2008/2009 im Auftrag des Bundeskriminalamts vom Sozialwissenschaftlichen FrauenForschungsInstitut Freiburg unter der Leitung von Prof. Dr. Cornelia Helfferich durch Prof. Dr. Barbara Kavemann, Ass. jur. Heike Rabe, M.A. Margit Wagner und der Mitarbeit von M.A. Heiko Hoffmann durchgeführt und 2010 veröffentlicht (Helfferich, Kavemann, Rabe 2010)

2 Unter Determinanten werden die Bedingungen und Voraussetzungen verstanden, die von subjektiver Bedeutung für die Betroffenen waren und aus ihrer Perspektive die Entscheidung für oder gegen eine Aussage bzw. Anzeige beeinflussten.

3 Ausbeutung stellte das Grundmuster aller Fälle dar und wird daher nicht gesondert aufgeführt.

4 Ausführlicher zur Methode der Untersuchung siehe Helfferich, Kavemann, Rabe 2010.

5 Eine Parallele findet sich in der Forschung zu Viktimisierungsprozessen bei häuslicher (Helfferich/ Kavemann/Lehmann 2004) und sexueller Gewalt (Kretschmann 1993).

\title{
Menschen - und Frauenhandel - welche Interventionen wären aus kriminologischer Sicht sinnvoll?
}

\author{
Weiterführend: Das Prostitutionsgesetz und seine Umsetzung, Ina Hunecke
}

Monika Frommel

Am 1.12.2009 moderierte Sandra Maischberger: „Sex als Ware: Sollten wir Prostitution verbieten? " < http://www.markenpost. de/bigimage.php?ID=86983>

Dokumentiert wurde - neben einem Beispiel der geglückten Umsetzung des ProstG - eine weit verbreitete Position, man könnte sie ein frauenpolitisches Credo nennen: Danach verkauft keine Frau ihren Körper freiwillig! Revidiert werden soll nach dieser besonders in Schweden konsensfähigen Position die Legalisierung der Prostitution durch das ProstG 2002. Wieso haben solche Appelle immer wieder ein Publikum? Betrachten wir also nach knapp einem Jahrzehnt die Geschichte dieser Reform.
1. Legalisierung der Prostitution, Verbot der „ausbeuterischen Prostitution“"

Erklärtes Ziel des 2002 geschaffenen ProstG (war die Verbesserung der rechtlichen Stellung von Prostituierten.1 Insbesondere sollte der Zugang zu den Sozialversicherungen ermöglicht bzw. erleichtert werden, was voraussetzt, dass die entsprechenden gewerberechtlichen Genehmigungen erteilt, Einnahmen versteuert werden, Arbeitsverträge geschlossen und der arbeits- und mietrechtliche Schutz aktiviert wird. Dies setzt aber eine Veränderung der gewerberechtlichen und bauordnungsrechtlichen Kontrollen voraus. Das Gewerberecht ist bis heute nicht geändert. Bis auf wenige Kommunen (Dortmund als bislang positives Beispiel, das aber im Jahr 2011 sein Modell beendete) blieb es allenfalls bei einer halbherzigen Legalisierung. Dies hat Folgen. Die strafrechtlichen Interventionen sind weitgehend weggefallen und stehen auch künftig nicht mehr zur Verfügung. Aber es gibt keine sinnvolle Alternativen. Weggefallen sind polizeiliche Kompetenzen, da die schlichte Förderung der Prostitution nicht mehr strafbar. Somit verbieten sich Razzien. Seit der Erweiterung der EU entfallen auch die früher üblichen ausländerrechtlichen Kontrollen. Sie konnten nur so lange benutzt werden als zahlreiche Prostitution noch nicht EU-Bürgerinnen waren. 